\title{
A Quick Generation Method for Power Grid Graphs and Models in SCADA System
}

\author{
Rongrong Cao \\ NARI Technology Development Co., Ltd, Nanjing, China \\ State Key Laboratory of Smart Grid Protection and Control, Nanjing, China
}

\begin{abstract}
In the large power grid dispatching and control system, the unmanned substations bring a large amount of graph drawing and model input work to the automation maintenance personnel. The complicated work increases the error probability and hidden dangers, and it is difficult to adapt to the development of the large power grid. Therefore, it is very necessary to realize the quick generation method of power grid graphs and models in the large power grid dispatching and control system. This paper proposes a method for quick generation of graphs and models in SCADA system. This method is based on the design concept of object-oriented graph and model integration and realizes the quick generation of similar substations. This design has been used in real application widely and makes the maintenance of the automation system more conveniently, rapidly and accurately.
\end{abstract}

Keywords-quick generation; graph drawing; model input; graph and model integration

\section{INTRODUCTION}

Supervisory Control and Data Acquisition (SCADA) system of the large power grid control center refers to the SCADA system for dispatching and control more than 50 unmanned substations [1]. In the process of generating the system, graph drawing and model input are required for each substation. The huge workload brings great pressure to the automation maintenance personnel. Therefore, how to speed up the generation of the graphs and models in the SCADA system and reduce the workload of the automation maintenance personnel has become a very important issue.

SCADA systems generally use the graph and model integration [2-5] technology to generate the graphs and models. Graph and model integration refers to the simultaneous input of model information into the database while drawing graphs, and it automatically establishes the relationship between the device information on the graph and the model information in the database. In the large power grid SCADA system, many substations have a lot in common in terms of graph and model information. In this way, repeated drawing of the same graph and frequent input of similar model information will occur during the system generation process, which wastes time and increases the probability of errors. In response to this situation, this paper proposes and designed a method that uses a similar substation as a template to automatically generate graphs and models of a new substation, and achieve the generation of graphs and models quickly and accurately.

\section{TRADITIONAL GRAPHS AND MODELS GENERATION METHOD}

The key to the generation of graphs and models in SCADA systems is graph and model integration technology. Graph and model integration is to generate the corresponding model information of the electrical graphic element in substation while the graphic element information is drawing. At the same time, the connection between the device graphic element and the database is realized, the power grid models are also generated, and the topology relationship is determined.

Using the graph and model integration technology, the graphs and models information of a substation is generated as shown in Figure. 1.

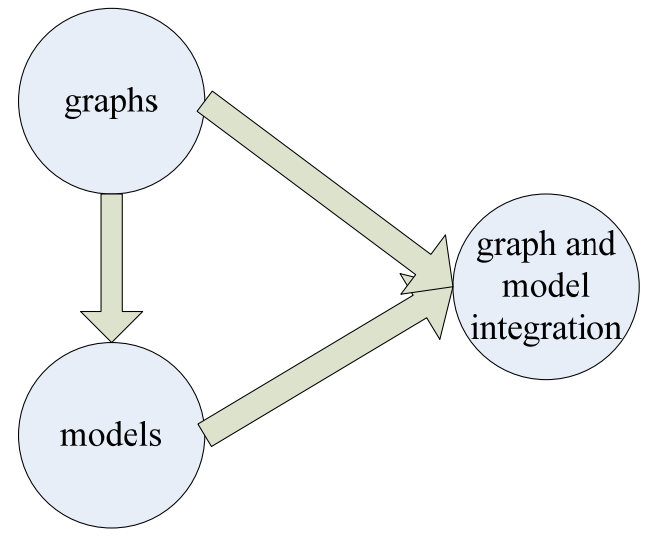

FIGURE I. GENERATION METHOD OF ONE SUBSTATION

Firstly, graphs information is generated by drawing graphic elements. Next, the models information of the graphic elements is input into the database. Based on the correlation of the two, the graph and model integration information of the substation is finally generated.

In the large power grid SCADA system, many substations are very similar in terms of graphs and models information. If you still use the traditional graphs and models generation method, the situation shown in Figure 2 will appear. 


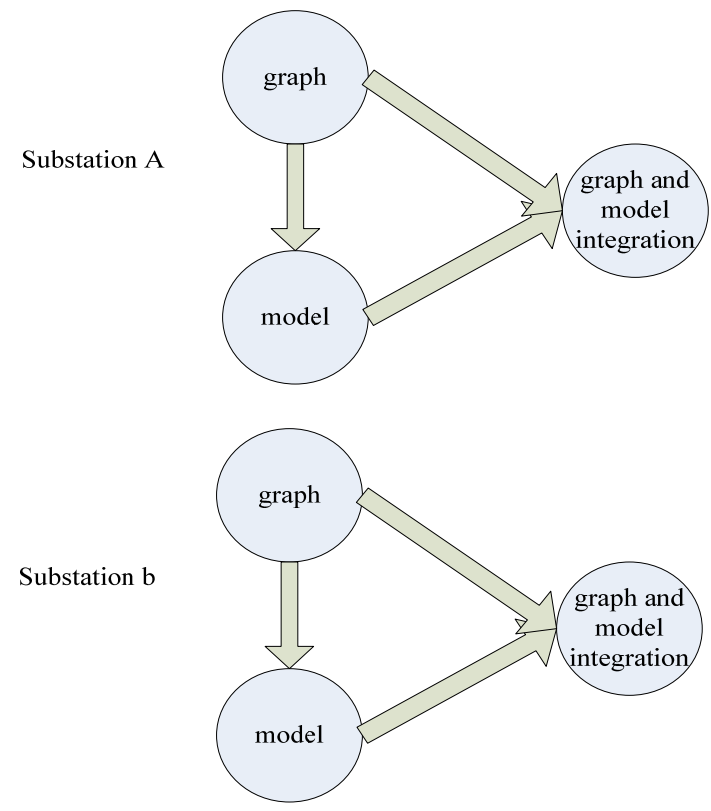

FIGURE II. TRADITIONAL GENERATION METHOD OF TWO SUBSTATIONS

It can be seen from Figure. 2 that although the graphs and models information of substation A and substation B is similar, they are still two separate parts in the generation process and cannot be reused. After the graphs and models information of the substation A is generated, the corresponding information of the substation B still needs to be regenerated. How to make use of the graphs and models information generated by the similar substations to reduce the repetitive operations in the graphs and models generation process has become the key to the quick generation of graphs and models in SCADA system.

\section{QUICK GENERATION METHOD}

\section{A. Design Principle}

Aiming at the similar characteristics of many substations in the large power grid SCADA system, a method for automatically generating a new substation graphs and models using a similar substation as a template was designed, which reduced the workload of automation maintenance personnel and implemented the graphs and models generation quickly and accurately. The specific principle is shown in Figure 3.
Substation A

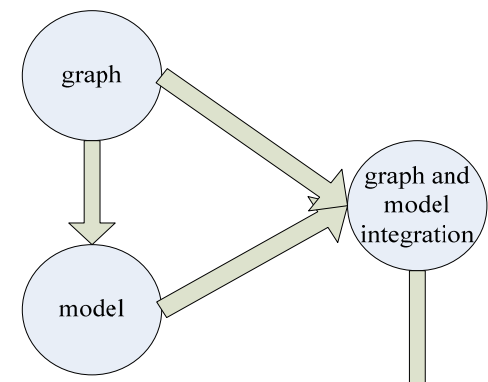

Substation b

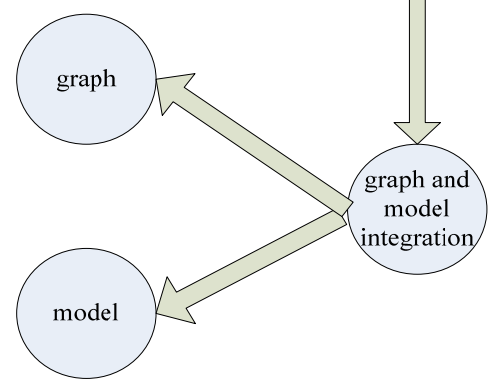

FIGURE III. QUICK GENERATION METHOD OF TWO SUBSTATIONS

Compared with Figure 2, it can be found in Figure 3 that the quick generation method of graphs and models can automatically generate the graphs and models information of the new substation based on the graph and model integration information generated by the similar substation. There is no need for manual operations such as graph drawing and model input, which greatly simplifies the job of generating the graphs and models, improves the efficiency, and reduces the probability of errors.

\section{B. Implementation}

The quick generation method of graphs and models can use existing substations as templates to automatically generate substations that are similar in graphs and models information. The substation template must be a substation that has already completed the graph drawing and model input work, and has finished a graph-model-related link. The new substation that needs to generate the graphs and models is similar to the template substation in terms of graphs and models information, such as the geometric shape, location of graphic elements, connection relationship between the graphic elements, the type of device, the naming rules, voltage level, and so on.

In the process of quick generation of the graphs and models, the substation template is used as a reference to automatically complete the drawing of the new substation graphs, and the model information of the new substation is automatically input, and then the graph information and model information of the new substation are automatically established the corresponding connection. In this way, the graphs and models of the new substation is completed quickly and conveniently. The basic flow is shown in Figure 4: 




FIGURE IV. FLOW CHART

\section{1) Generating graphs automatically}

Based on the graphs of the substation template, the graphs information of the new substation is automatically generated and what you see is what you get.

Graphs are composed of graphic elements. Therefore, the key to the automatic generation of graphs is the automatic generation of graphic elements.

The content that each graphic element needs to be generated includes the basic information of the graphic element in the system and the shape information that the graphic element needs when drawing. The shape information is divided into two parts: commonality and personality. In addition to the basic information and shape information, the device graphic element also needs to generate connection information with other graphic elements.

The basic information of the graphic element generally includes the type, id number, application type, display type, and so on. The information has nothing to do with the external shape and it is a set of attributes that need to be set in the system.

The common part in the shape information refers to the attributes of each type of graphic elements in terms of its shape.
It is generally the information related to drawing, includes the largest bounding box of the graphic element, its own plane, its own level, line type, line color, line width, fill mode, fill color, and rotation angles, etc.

The personality part of the shape information of the graphic element is different according to the type of each element, such as a linear element, its own attributes include the starting point, the ending point, and the shape and size of the arrow at the beginning and end, etc. The shape information is needed while drawing this type of graphic element.

If it is a device graphic element, its graphic element information should also include connection information, generally the maximum number of pin, the connection status of each pin, and the connection relationship between the graphic elements.

It should be noted that the display textual elements of the substation name and some of the flags call links require special handling and automatic replacement according to the input substation name.

After the graphic elements of the new substation are generated, the graphs of the new substation are automatically generated.

\section{2) Generating models automatically}

The automatic generation of the models is mainly the automatic generation of the database information of each device. According to the graph and model integration technology, first obtain the model information of the corresponding device graphic element from the substation template, and then automatically generate the model information of the new substation, and finally complete the automatic generation of the new substation models.

Based on the device graphic element of the substation template, the model information of the new substation is automatically generated. Most of the content is similar, such as the name, voltage level, and its application, etc., and it is necessary to replace the substation information of the devices.

After the bay information is automatically generated, it also needs to search internal devices, automatically generate internal device information according to the naming rules, and pay attention to the setting of the bay information to which the device belongs.

Some triggered devices, such as transformer winding elements, are generated by a transformer trigger. When automatically generating new device model information, it is necessary to create transformer information first and then trigger the corresponding winding information.

The AC line end is also triggered by the AC line segment, and there are large differences in the AC line segments of different substations. Special processing is also required to provide such as input methods and then they are automatically generated as needed.

3) Associating of graphs and models automatically

When the graphs and models information is automatically generated, the last thing to do is to associate them. Each model in the database has a unique identifier key ID. Each graphic 
element also has a unique identifier obj ID. When the two are associated with each other, the model information in the database corresponds to the device graphic element on the graphs, as shown in Figure 5.

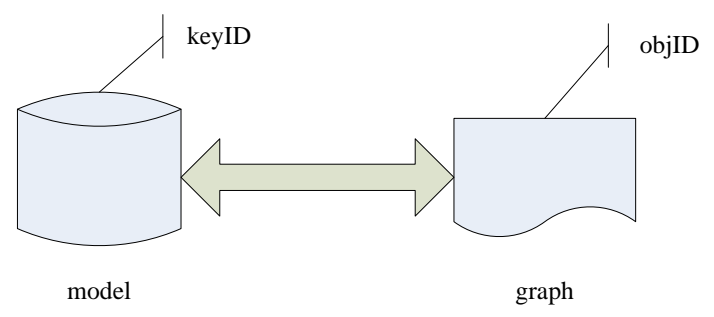

FIGURE V. AUTOMATIC ASSOCIATION

After the graphs and models information is linked, the generation of graphs and models of the entire substation is completed.

\section{CONCLUSION}

The quick generation method of the graphs and models has been widely used in systems such as Shenzhen SCADA system, and it has achieved good results in preventing misoperation and reliability monitoring and has brought great convenience to the engineering and maintenance of SCADA system.

- More convenient of engineering work. This method reduces a lot of duplicate drawing and input work, speeds up system generation. For example, in Shenzhen, the generation of graphs and models in a common $110 \mathrm{kV}$ substation in the traditional way, skilled operators need at least ten minutes. According to the quick generation method, it only takes a few minutes and the work effectiveness is greatly improved.

- More accurate of engineering work. Graphs and models are automatically generated and associated, reducing human error.

- Faster maintenance work. This method generates the graphs and models in system quickly and automatically and simplifies the maintenance work.

- Safer maintenance work. This method ensures the correctness of maintenance work and eliminates potential safety hazards.

\section{REFERENCES}

[1] ZHAO Weijun, SHI Wenjiang, WANG Rongmao, WANG Hongzhe, "A Bay Map Automatic Generation Method for Big Computer Centralized SCADA System in Unmanned Substations," Automation of Electric Power Systems, vol 32, no 13, pp. 53-57, July 2008.

[2] XU Ai-chun, WEI Yan-hua, ZHANG Jian-min, "Design and implementation of vector graphics editing system based on CIM/SVG," RELAY, vol 34,no 23, pp. 49-53, Dec 2006.

[3] Shao Lidong, Wu Wenchuan, Zhang Boming, "A CIM-BASED INTERACTIVE GRAPHICS SYSTEM FOR EMS/DMS,” Automation of Electric Power Systems, vol 27,no 20, pp.11-15, Oct 2003.

[4] DONG Zhao-xia, DAI Qi, YANG Feng, "Power Network Modeling Method Based on CIM and SVG," Proceedings of the CSU-EPSA, vol 18,no 5, pp. 58-61, Oct 2006.
[5] HE Rui, MIAO Xuefan, CHEN Jianyun, "Design and Development of A Software of Drawing SCADA's Monitoring Graphics Based on SVG” Industrial control computer, vol 19,no 6, pp. 32-33, Sep 2006. 